\title{
Pregnancy complicated by influenza A ARDS requiring consecutive VV- ECMO treatment with successful vaginal delivery
}

\author{
Peter Radsel ${ }^{1}$ - Vojka Gorjup ${ }^{1} \cdot$ Anja Jazbec ${ }^{1} \cdot$ Rihard Knafelj $^{1} \cdot$ Miha Lucovnik $^{2} \cdot$ Gorazd Kavsek $^{2}$. \\ Lilijana Kornhauser Cerar ${ }^{2} \cdot$ Marko Noc $^{1}$
}

Received: 2 March 2018 / Accepted: 14 May 2018 / Published online: 17 May 2018

(c) The Japanese Society for Artificial Organs 2018

\begin{abstract}
A 29-year-old woman presented with influenza A ARDS at $23^{+0}$ weeks of gestation. Mechanical ventilation failed and VVECMO was started in a non-ECMO hospital. Transportation was performed on ECMO. Within 5 days ECMO weaning was successful. Fetal condition was stable, and decision to continue pregnancy was taken. However, second VV-ECMO was needed due to ventilator-associated pneumonia. At $25^{+6}$ weeks, the patient spontaneously delivered a neonate vaginally. Patient's condition improved, and ECMO could be removed 10 days postpartum. 2-year follow-up showed no severe consequences in the mother and the child.
\end{abstract}

Keywords Influenza A $\cdot$ ARDS $\cdot$ VV-ECMO $\cdot$ Pregnancy $\cdot$ Vaginal delivery

\section{Introduction}

In 2009, H1N1 pandemic pregnant women were 7 times more likely to be hospitalized and 4 times more likely to be admitted to ICU than non-pregnant women [1]. At risk were mostly women in second and third trimester [2, 3]. Immunological changes increased ventilatory demand, decreased lung functional residual capacity, and decreased oncotic pressure, all constitute predisposing factors for respiratory complications [4]. Mortality in pregnant women with influenza who require ICU treatment ranges from 8 to $18 \%$ mostly due to pulmonary complications [2, 4]. In 2009, outbreak mechanical ventilation failed in several occasions and since then veno-venous extracorporeal membrane oxygenation (VV-ECMO) has been used as a rescue therapy. The literature shows $75 \%$ survival of pregnant women treated with extracorporeal life support with fetal survival of 65\% [5].

Peter Radsel

pradsel@gmail.com

1 Department of Intensive Internal Medicine, University Medical Center Ljubljana, Ljubljana, Slovenia

2 Division of Gynecology and Obstetrics, Department of Perinatology, University Medical Center Ljubljana, Ljubljana, Slovenia
We report a gravid patient with ARDS secondary to influenza infection requiring ECMO support. Insertion was performed at a non-ECMO hospital. After successful ECMO weaning, ventilator-associated pneumonia resulted in second ECMO insertion. She delivered vaginally without complications, while on VV-ECMO with excellent maternal and neonatal long-term result.

Presented case raises many unanswered questions concerning the management of critically ill pregnant women.

\section{Case presentation}

A 29-year-old woman with no medical history and uneventful first pregnancy presented to gynecologist at $23^{+0}$ weeks of pregnancy with a 3-day history of fever and cough. On examination, she was febrile and tachypneic with $70 \%$ arterial saturation. She was transferred to emergency department. Chest X-ray showed diffuse lung infiltrates and laboratory results (CPR $166 \mathrm{mg} / \mathrm{L}$, PCT $2.3 \mu \mathrm{g} / \mathrm{L}$ ) were consistent with pneumonia. Due to progressing respiratory failure she was admitted to ICU at a tertiary hospital.

Antibiotic treatment for pneumonia during influenza epidemic was chosen-azithromycin, ceftriaxone, and oseltamivir. Trial of non-invasive ventilation was unsuccessful. She was mechanically ventilated with 


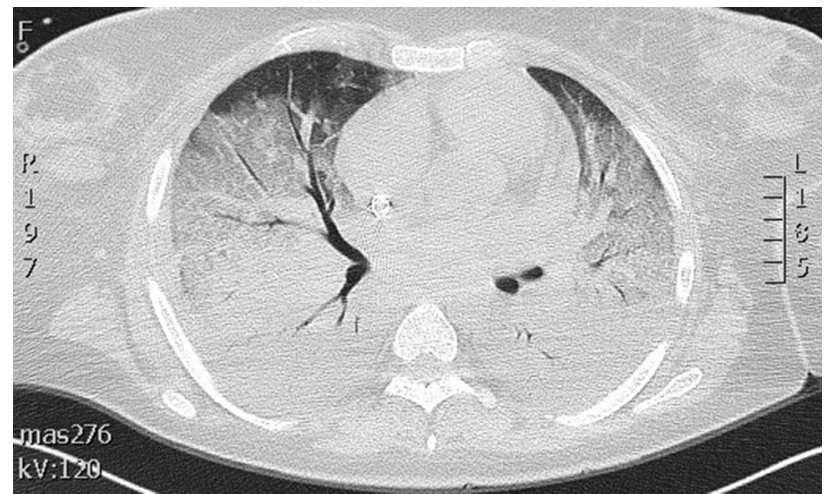

Fig. 1 Lung CT showed bilateral diffuse lung consolidation

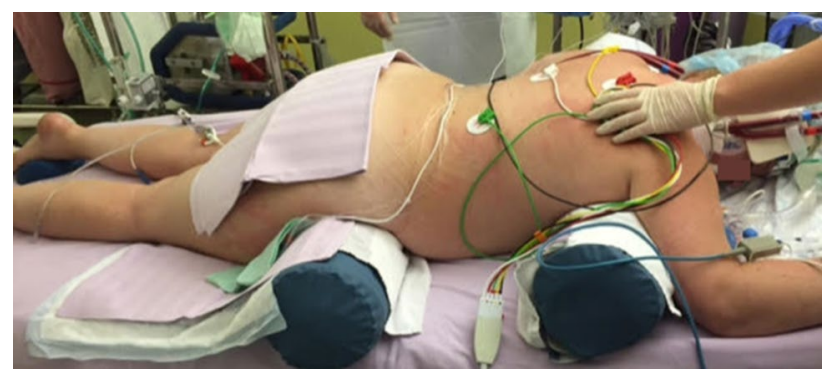

Fig. 2 Roller pillows were used under upper thorax and groin to optimize prone position

100\% oxygen and high PEEP. Her condition temporarily improved, but within hours her respiratory function declined again and prone ventilation was implemented. ECMO center was consulted 2 days later. On pressure-control ventilation ( $\mathrm{P}$ insp $35 \mathrm{~cm} \mathrm{H}_{2} \mathrm{O}$, PEEP 15, $\mathrm{FiO}_{2} 70 \%$ ), her arterial $p \mathrm{O}_{2}$ was $7.9 \mathrm{kPa}$ and $p \mathrm{CO}_{2} 6.4 \mathrm{kPa}$. Transport in such condition was considered to be too risky and a decision to send a team to insert VV ECMO was taken. Our strategy was to insert the inflow cannula through right femoral vein and the outflow cannula through right jugular vein. Central venous catheter used for sedation had already been in place in the right femoral vein; therefore, left femoral vein puncture was done. After having problems with wire passing to the atrium, a dual-lumen 27Fr cannula (Avalon Elite ${ }^{\circledR}$ ) was inserted via right jugular vein. The procedure was guided with transesophageal ultrasound. Institution of ECMO (Maquet Cardiohelp ${ }^{\circledR}$ ) resulted in immediate improvement in oxygenation, and lung-protective ventilation could be initiated. The patient was transferred to ECMO center.

Influenza A RT-PCR assay was positive from pharyngeal smear. Lung CT was consistent with ARDS (Fig. 1). Prone position was used while on ECMO (Fig. 2). Fetal ultrasound showed appropriate weight for gestational age and no morphologic abnormalities. After discussion with patient's family, a multidisciplinary decision to continue pregnancy was reached.

The therapy resulted in improvement of infection and lung function. On the 5th day ECMO was removed. 3 days later, the patient became febrile and chest X-ray showed new lung consolidations. Antibiotic therapy was changed although no bacterial pathogen was isolated. Severe hypoxemia developed again, and decision for new VV-ECMO insertion was made. Venous ultrasound showed right jugular vein thrombosis. Having problems with left femoral cannulation at the first attempt, we decided to insert cannulas under fluoroscopy guidance. Inserting the wire to the right atrium from left femoral vein failed again. Angiography showed left iliac vein malformation that prevented cannula insertion. Cannulas were inserted through right femoral and left jugular vein. In the following days, attempts to stop sedation failed due to restlessness and tachypnea. Different analgesic and sedation protocols were used including sevoflurane. Despite antibiotic therapy and no obvious non-pulmonary site of infection, inflammation markers remained high. We changed cefepim to imipenem/cilastatin, vancomycin, and amphotericin B. Fetal condition was assessed regularly and remained stable. Betamethasone was given for lung maturation at 24 weeks.

During morning care on the 7th day of VV-ECMO treatment $\left(25^{+4}\right.$ weeks of gestation) cervical mucus plug was discharged. An obstetrician diagnosed onset of labor with regular uterine contractions and advanced cervical dilatation. Due to cephalic presentation and good fetal condition, decision to deliver vaginally was reached. Labor was augmented with oxytocin $(5 \mathrm{mU} / \mathrm{min})$. Within $2 \mathrm{~h}$, a female neonate with birth weight of $820 \mathrm{~g}$ was born and was immediately transferred to neonatal ICU. Apgar scores were 6 at 1 min and 8 at 5 min and umbilical artery pH was 7.27. Third stage of labor was managed with uterotonics $(5 \mathrm{U}$ oxytocin IV after delivery followed by $40 \mathrm{mU} / \mathrm{min}$ infusion and $0.2 \mathrm{mg}$ methylergometrine IM) and controlled cord traction. The mother received protamine sulfate to prevent postpartum hemorrhage. Intact placenta was delivered within $20 \mathrm{~min}$ and overall blood loss was $<500 \mathrm{~mL}$.

After delivery, the patient's condition improved. ECMO was removed on day 10 . Weaning from mechanical ventilation was complicated by delirium and critical illness myopathy. After performing percutaneous tracheostomy, spontaneous breathing recovered. She was transferred to perinatology department and later to rehabilitation center. Chest X-ray after discharge showed almost complete lung restitution.

Due to severe respiratory distress, the neonate was intubated and received surfactant (Curosurf $\left.{ }^{\circledR}, 120 \mathrm{mg}\right)$. Highfrequency oscillation ventilation (HFOF) was instituted. Pulmonary hypertension was treated with inhalatory NO. HFOV was needed for 3 weeks and intermittent positive pressure ventilation until day 32. After successful extubation, the 
infant needed non-invasive support until day 86. As signs of bronchopulmonary dysplasia had developed, she received three courses of dexamethasone. Hyperirritability observed in the first 2 weeks, and treatment with phenobarbital was attributed to maternal drugs transferred to the fetus. The infant was discharged at 3 months and needed addition of oxygen delivered by nasal cannula. It was discontinued at the chronologic age of 14 months. At 24-month follow-up, no obvious neurological deficit was identified.

\section{Discussion}

Although VV-ECMO has become a routine treatment of ARDS in pregnant women still many dilemmas remain. Respiratory insufficiency can develop within hours. Such patients commonly present to hospitals without facilities for ARDS treatment such as prone ventilation, $\mathrm{CO}_{2}$ removal devices, or VV-ECMO. Whether we should transport such patients prior to ECMO implantation to larger centers or plan a delayed transportation while already stabilized on ECMO remains an individual decision. We did not encounter difficulties during road transportation, and there are similar cases reported in the literature [6] although such data are biased.

Already in the non-ECMO hospital prone position was used as a trial to improve ventilation. Although there are limited data on the use of prone position in pregnancy [7], we continued with intermittent prone position also during ECMO treatment (Fig. 2). Lower abdominal pressure was measured regularly and it remained under $12 \mathrm{mmHg}$.

The biggest dilemma is whether to terminate pregnancy or wait if the fetus is extremely premature. Physiologically, we would expect improvement of lung mechanics postpartum and faster maternal improvement, but data in the literature do not support this assumption [8-10]. Therefore, our decision was not to terminate the pregnancy given the uncertain benefit for the mother and high risks of neonatal death and long-term disability at 23 weeks of gestation [11]. Instead, we decided to keep the patient pregnant since even small delays in delivery provide significant reductions of neonatal risks [11].

To reduce the thrombo-embolic and hemorrhagic complications during VV-ECMO treatment [12], a strict protocol for anticoagulation was chosen. Activated partial thromboplastin time (aPTT) was measured several times daily with target values 50-60 s and anti-factor Xa for unfractionated heparin (UFH) once daily (target $0.3-0.5 \mathrm{IU} / \mathrm{mL}$ ). Despite high doses of UFH (up to $3000 \mathrm{IU} / \mathrm{h}$ ), we could not achieve adequate anticoagulation. Patient's aPTT levels were 40-50 s and anti-factor Xa 0.2-0.3 IU/mL. This may be one of the factors that the patient developed vein thrombosis at the site of first cannula. As right jugular vein was not available for second cannulation, we decided for bi-femoral approach under fluoroscopic guidance. Keeping in mind potential harm for the fetus, we limited fluoroscopy time to be under $10 \mathrm{~s}$, aiming mostly to the right atrium to see wire and cannula tips. Having problems with wire advancing in the left femoral vein, we performed angiography with only $10 \mathrm{~mL}$ of contrast. Failing with left femoral approach, we ended up with right femoral and left jugular cannulas. This could have been our first approach, but our experience with left jugular cannulation was limited. After delivery, the patients received therapeutic low molecular heparin with no complications.

When labor began, we opted for vaginal delivery, since maternal and neonatal risks of cesarean delivery at that moment outweighed the risks associated with vaginal delivery. Obstetric and neonatal teams were at the bedside, and equipment for neonatal transport was available. Active management of the third stage of labor together with protamine sulfate to reverse the effects of UFH has been successfully employed to prevent postpartum hemorrhage.

Vaginal delivery was quick and uneventful. As far as we know, this is the second case of successful vaginal delivery during ECMO support reported [13]. There are more cases of cesarean section on ECMO published [14, 15], and although this allows more rapid delivery in the critically ill women, it increases the risk of postoperative complications and has been associated with higher maternal mortality [16].

Prompt recovery of maternal condition after delivery is in contrast with data on maternal outcomes in pregnant patients with ARDS on VV ECMO [8-10]. We believe more research is needed on effects of pregnancy in critically ill patients.

Lastly, during intensive care treatment, the fetus is exposed to drugs that can influence its development. In our case, sedation withdrawal attempts resulted in severe tachypnea and inspiratory transpulmonary pressure of $35 \mathrm{cmH}_{2} \mathrm{O}$. To prevent further lung injury, we used sedation and analgesia which ended in severe dependence and delirium in the recovery phase. Therefore, we used also sedatives such as sevoflurane with limited experiences in pregnancy. None of these treatments seemed to have a fetotoxic effect since neonatal postpartum course was comparable to that of other neonates born at such gestations. It is, however, impossible to estimate the potential teratogenic effects of these treatments as they have been given to the mother at 23/24 weeks, when organogenesis is largely complete.

\section{Conclusion}

Many dilemmas on how to treat pregnant women with severe ARDS still exist, and maternal and fetal mortality is high. Nevertheless, teamwork can lead to fascinating cases with excellent long-term results. 
Acknowledgements Medical staff at Department of Intensive Internal Medicine and Department of Gynecology and Obstetrics, UMC Ljubljana.

\section{References}

1. Creanga AA, Johnson TF, Graitcer SB, et al. Severity of 2009 pandemic influenza $A(\mathrm{~N} 1 \mathrm{~N} 1)$ virus infection in pregnant women: New York City, May-June 2009. Obstet Gynecol. 2010;115:717-26.

2. Louie JK, Acosta M, Jamieson DJ, et al. Severe 2009 H1N1 influenza in pregnant and postpartum women in California. NEJM. 2012;362:27-35.

3. ANZIC Influenza Investigators and Australasian Maternity Outcomes Surveillance System. Critical illness due to 2009 A/H1N1 influenza in pregnant and postpartum women: population based cohort study. BMJ 2010;340:c1279.

4. Jamieson DJ, Honein MA, Rasmussen SA, et al. H1N1 2009 influenza virus infection during pregnancy in the USA. Lancet. 2009;374:451-8

5. Moore SA, Dietl CA, Coleman DM. Extracorporeal life support during pregnancy. J Thorac Cardiovasc Surg. 2016;151:1154-60.

6. King PT, Rosalion A, McMillan J, Buist M, et al. Extracorporeal membrane oxygenation in pregnancy. Lancet 2000;356:45-6.

7. Samanta S, Samanta S, Wig J, Baronia AK. How safe is the prone position in acute respiratory distress syndrome at late pregnancy? Am J Emerg Med. 2014;32:687.
8. Collop NA, Sahn SA. Critical illness in pregnancy. An analysis of 20 patients admitted to a medical intensive care unit. Chest. 1993;103:1548-52.

9. Tomlinson MW, Caruthers TJ, Whitty JE, et al. Does delivery improve maternal condition in the respiratory-compromised gravida? Obstet Gynecol 1998;91:108 -11.

10. Catanzarite V, Willms D, Wong D, et al. Acute respiratory distress syndrome in pregnancy and the puerperium: causes, courses, and outcomes. Obstet Gynecol. 2001;97:760-4.

11. Tyson JE, Parikh NA, Langer J, et al. Intensive care for extreme prematurity: moving beyond gestational age. N Engl J Med. 2008;358:1672-81.

12. Nair P, Davies AR, Beca J, et al. Extracorporeal membrane oxygenation for severe ARDS in pregnant and postpartum women during the $2009 \mathrm{H} 1 \mathrm{~N} 1$ pandemic. Intensive Care Med. 2011;37:648-54.

13. Kunstyr J, Lips M, Belohlavek J, et al. Spontaneous delivery during veno-venous extracorporeal membrane oxygenation in swine influenza-related acute respiratory failure. Acta Anaesthesiol Scand. 2010;54:1154-5.

14. Panarello G, D'Ancona G, Capitanio G, et al. Cesarean section during ECMO support. Minerva Anestesiol. 2011;77:654-7.

15. Łysenko L, Zaleska-Dorobisz U, Blok R, et al. A successful cesarean section in a pregnant woman with A (H1N1) influenza requiring ECMO support. Kardiochir Torakochirurgia Pol. 2014;11:216-9.

16. Jenkins TM, Troiano NH, Graves CR, et al. Mechanical ventilation in an obstetric population: characteristics and delivery rates. Am J Obstet Gynecol. 2003;188:549-52. 